\title{
On the development of radiation tolerant surveillance camera from consumer-grade components
}

\author{
Ambrožič Klemen ${ }^{1, *}$, Snoj Luka ${ }^{1, * *}$, Öhlin Lars ${ }^{2, * \star *}$, Gunnarsson Jan ${ }^{2, \star * \star *}$, and Barringer \\ Niklas $^{2, \dagger}$ \\ ${ }^{1}$ Jožef Stefan Institute, Jamova cesta 39, SI-1000 Ljubljana, Slovenia \\ ${ }^{2}$ ISEC-Industrial Security AB , Diabasgatan 12, SE-254 68 Helsingborg, Sweden
}

\begin{abstract}
In this paper an overview on the process of designing a radiation tolerant surveillance camera from consumer grade components and commercially available particle shielding materials is given. This involves utilization of Monte-Carlo particle transport code MCNP6 and ENDF/B-VII.0 nuclear data libraries, as well as testing the physical electrical systems against $\gamma$ radiation, utilizing JSI TRIGA mk. II fuel elements as a $\gamma$-ray sources. A new, aluminum, $20 \mathrm{~cm} \times 20 \mathrm{~cm} \times 30 \mathrm{~cm}$ irradiation facility with electrical power and signal wire guide-tube to the reactor platform, was designed and constructed and used for irradiation of large electronic and optical components assemblies with activated fuel elements. Electronic components to be used in the camera were tested against $\gamma$-radiation in an independent manner, to determine their radiation tolerance. Several camera designs were proposed and simulated using MCNP, to determine incident particle and dose attenuation factors. Data obtained from the measurements and MCNP simulations will be used to finalize the design of 3 surveillance camera models, with different radiation tolerances.
\end{abstract}

\section{Introduction}

In order to maintain video surveillance in radiation environment, the surveillance cameras have to be radiation tolerant. This can be achieved by selecting radiation tolerant (rad hard) camera components, or by adequate shielding of non rad hard components. This paper describes development of a camera, constructed from consumer grade electronic and optics components with the aid of publicly available rad-hard component databases [1] and a camera shielding enclosure design. Such a camera would be used inside NPP containment, spent nuclear fuel and waste facilities, in medical radiology, etc., and would therefore be exposed to both neutrons and $\gamma$-rays. Since consumer-grade parts usually do not exhibit heightened radiation tolerance, they have to be shielded from radiation.

The end result is to be 3 distinct surveillance camera models, with different levels of incident radiation tolerance requirements, supplied by ISEC. These levels were determined by close cooperation of ISEC and their customers and market demands and are given in terms of ${ }^{60} \mathrm{Co}$ dose in table 1 .

\footnotetext{
*e-mail: klemen.ambrozic@ijs.si

** e-mail: luka.snoj@ijs.si

*** e-mail: laoh@isec.se

**** e-mail: jagu@isec.se

†e-mail: niba@isec.se
} 
Table 1: Radiation tolerance goals for three different camera model versions

\begin{tabular}{lll}
\hline Camera model version & Dose-rate $\left[\mathrm{Gy} \mathrm{h}^{-1}\right]$ & Cumulative dose [Gy] \\
\hline High radiation tolerance & $10^{3}$ & $10^{5}$ \\
Medium radiation tolerance & $10^{3}$ & $10^{4}$ \\
Low radiation tolerance & $10^{1}$ & $10^{3}$ \\
\hline
\end{tabular}

Since radiation tolerance is not a required feature in consumer grade electronics, no data on radiation resistance is provided by the manufacturer and very little work on radiation tolerance assurance has been provided by third parties, due to low volume requirements of such components and consequently high production costs. Therefore the components to be used in the camera have to be tested against radiation tolerance, and their operational parameters monitored during the tests. This way a list of components radiation tolerances can be made. The most sensitive components or modules are then replaced by their rad hard counterparts or shielding requirements readjusted to most sensitive components. Strategical placement of components and modules inside shielding enclosure has a significant impact on overall radiation tolerance of the camera system.

The most appropriate tool for shielding design and analysis of doses are Monte Carlo neutron and photon transport codes, such as MCNP [2]. In the paper the above mentioned activities are presented.

\section{Irradiation tests}

Initially, camera modules, electronic and optics assemblies and components intended to be used in the camera models, were checked for their rad-hard counterparts in the available databases [1], and tested against radiation. The purpose of these tests is to determine the doses (both neutron and $\gamma$ ) that the components and modules can withstand. These tests were performed at the JSI TRIGA mark II reactor [3] (TRIGA) in the existing and newly constructed irradiation facilities. A gas-flow ionization chamber in close proximity of the test assembly was used for cumulative dose and dose-rate measurements during the irradiation procedure. A custom assembly-parameter-reader was constructed for each test assembly, which monitored several aspects of the assembly operation. A failure or malfunction would be noted on the parameter reader log, thus determining the radiation tolerance of the component.

Activated TRIGA fuel elements were utilized as a $\gamma$-ray source, either in an in-core Triangular channel (TriC) irradiation facility, or ex-core, by moving up to 6 TRIGA fuel elements to the fuel element rack on the edge of the reactor tank [4]. A new irradiation facility, an aluminum box with internal dimensions of $20 \mathrm{~cm} \times 20 \mathrm{~cm} \times 30 \mathrm{~cm}$ and a cable guide tube, leading to the reactor platform, was designed and constructed to utilize ex-core fuel elements as $\gamma$-ray sources. After the irradiations the logged data for both ionization chamber and for electronic components were evaluated and points of failure for each component determined. This process was repeated several times over, using different components and module designs, until a satisfactory radiation tolerance of the module was obtained. After the irradiations, the measurement data were post-processed, and points of failure determined. An example of an irradiation test data visualization is presented in figure 1 .

\section{Computational analysis}

Monte Carlo particle transport calculations were carried out in order to assist in the development of a suitable shielding enclosure of the camera, as well as proposing additional shielding of the individual components. Several different stages of modeling the shielding enclosure took place, from scoping calculations on block shielding materials to progressively more complex geometrical models towards 


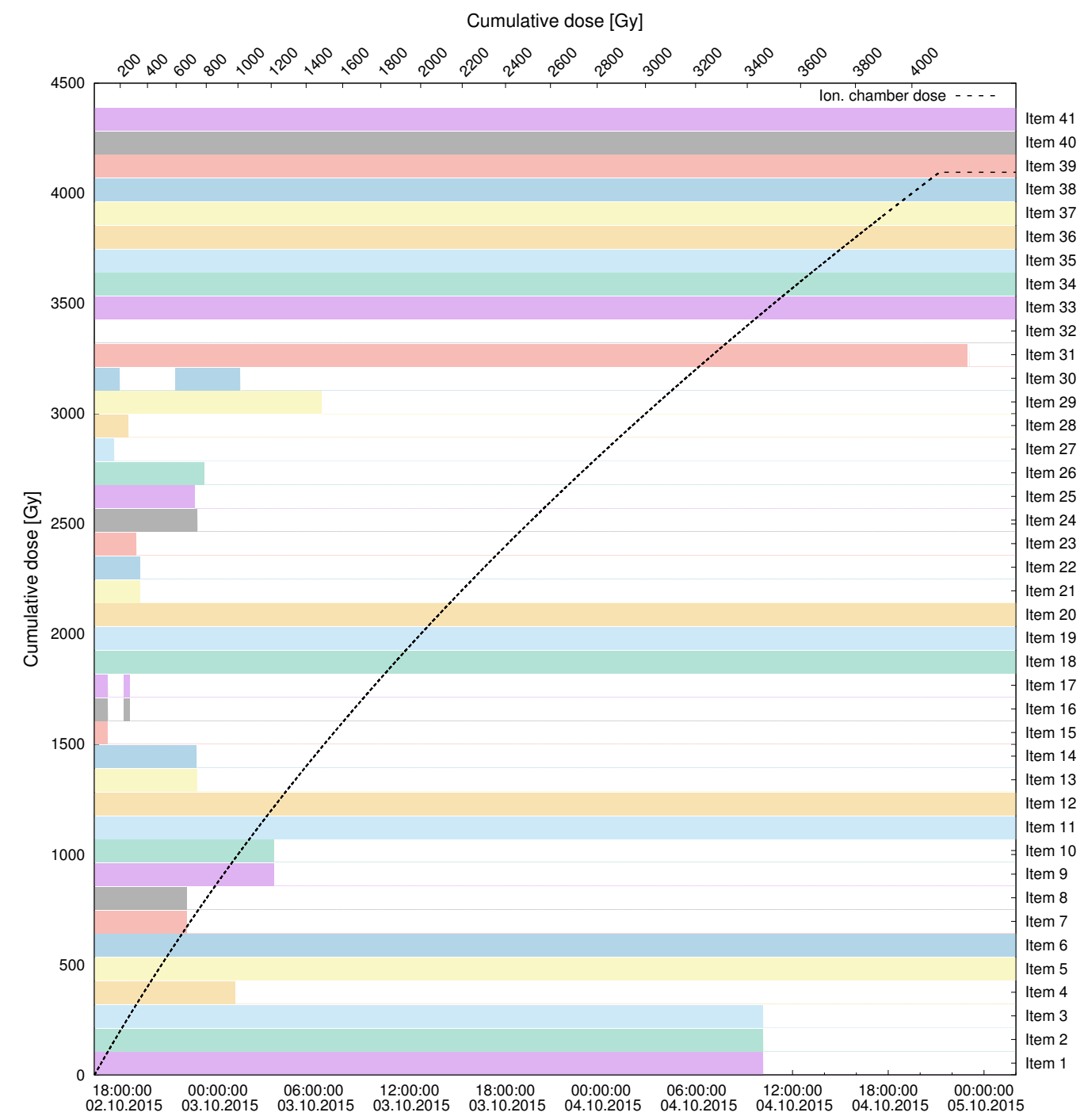

Figure 1: An example of electronic componets test data, with test items in the horizontal rows, and ionization chamber measurement data. Colored row means, the item is operational.

a final design. Several different neutron and photon spectra were used for radiation sources in the simulations (fig. 3). Photon spectra were previously calculated [5], and $6 \mathrm{MeV}$ and $7 \mathrm{MeV} \gamma$ lines added, to represent a percentage of whole spectrum, to simulate water activation $\gamma$ lines.

Real world scenarios are taken into account in terms of radiation source geometries, source particle types and spectra. Two plane sources, one with particle emission directly towards the camera (fig. 2a), one with isotropic emission over half the solid angle (fig. 2b) which are representative of point sources at distance and close proximity, a half spherical (fig. 2c) and a spherical source (fig. 2d), with isotropic particle emission from the half sphere / sphere surface over half the solid angle inwards, 
which are representative of uniformly distributed radiation sources throughout the space, and account for backscattering from walls, should the camera be positioned close to the wall.

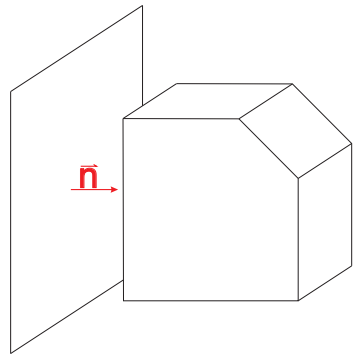

(a) Plane direct source geometry.
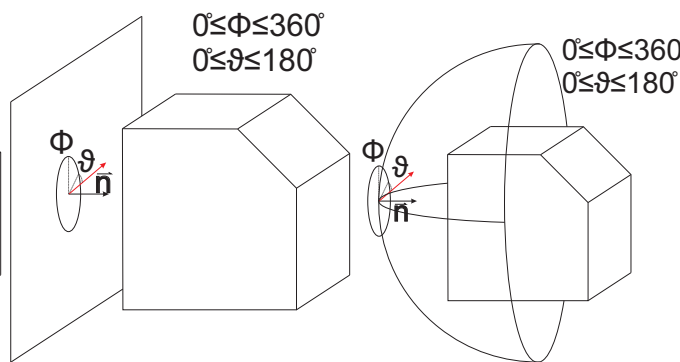

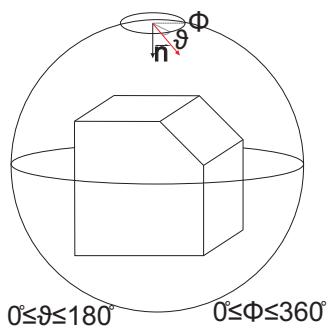

$0 \leq \vartheta \leq 180^{\circ}$ (c) Isotropic source geome- (d) Isotropic source over try over half sphere shell in- sphere shell inwards. wards.

Figure 2: Source geometries, used for simulations.

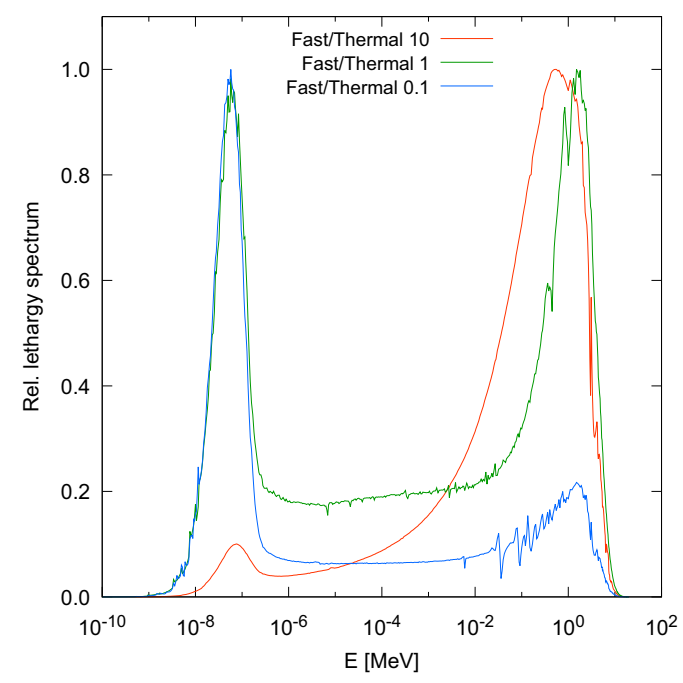

(a) Source neutron lethargy spectra, used in simulations.

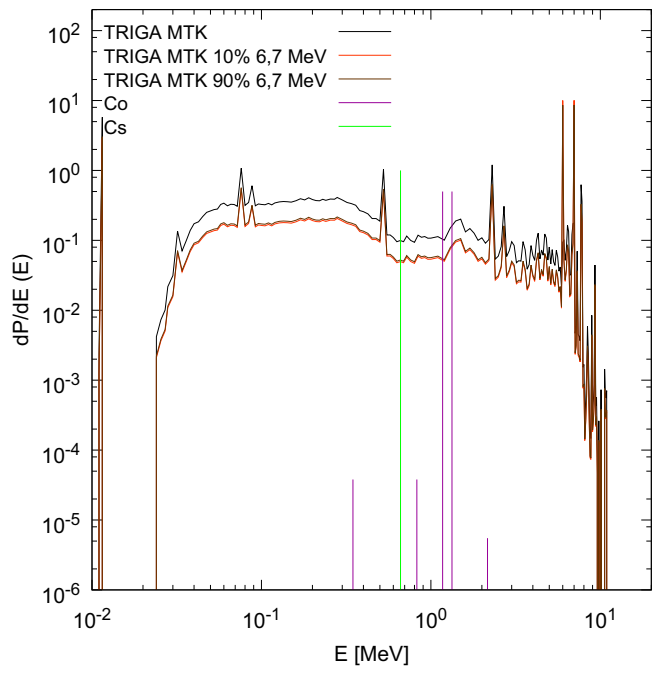

(b) Source $\gamma$-ray spectra used in simulations.

Figure 3: Source particle spectra, used for simulations.

Several different spectra, for both neutron and $\gamma$ rays were used in simulations. Neutron spectra are of ${ }^{235} \mathrm{U}$ fission type, given in terms of ratios between fast $\left(E_{N}>1 \mathrm{keV}\right)$ and thermal $\left(E_{N}<0.625 \mathrm{eV}\right)$ neutron flux $\frac{F_{N}}{T h_{N}}$ and aim to represent current reactor spectra for thermal $\left(\frac{F_{N}}{T h_{N}}=0.1\right)$ and fast $\left(\frac{F_{N}}{T h_{N}}=1\right)$ fission reactors, as well as fusion devices $\left(\frac{F_{N}}{T h_{N}}=10\right) \cdot \gamma$-ray spectra are spectra calculated inside TRIGA irradiation facilities, modified TRIGA irradiation facilities spectra (MTK: minor thermalization column), with added $6 \mathrm{MeV}$ and $7 \mathrm{MeV} \gamma$ lines arising from primary water activation (percentage denotes spectral fraction of $6 \mathrm{MeV}$ and $7 \mathrm{MeV}$ lines) due to (n,p) reactions on ${ }^{16} \mathrm{O}$, and ${ }^{60} \mathrm{Co}$ and ${ }^{137} \mathrm{Cs}$ spectra.

Doses were calculated using either flux-to-dose conversion factors (ICRP-21 H*10 [6] for $\gamma$-rays, 
ICRP-74 H*10 [7] for neutrons and ASTM E-722 [8] for neutron dose on silicon)(fig. 4), or direct kerma computations using MCNP track-length heating estimate (F6 tally in MCNP) [2].

Shielding material compositions data-sheets were supplied by the shielding manufacturers and isotopic compositions evaluated using MATSSF [9] computer code.

ENDF/B-VII.0 nuclear data libraries [10] were used in the calculations.

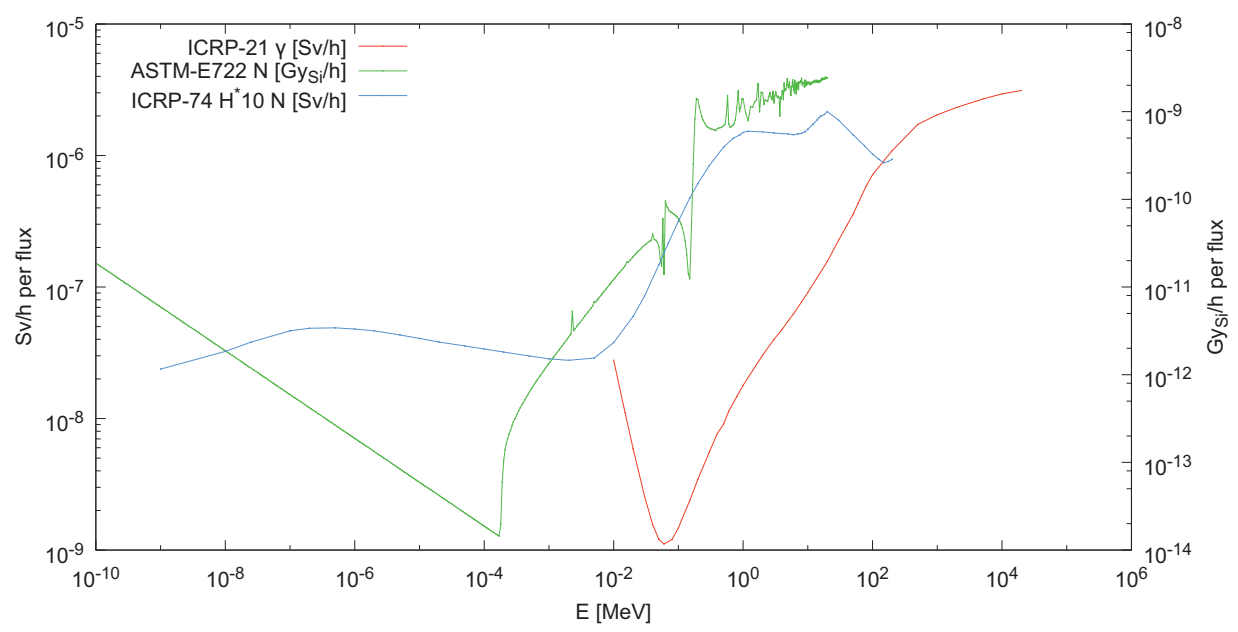

Figure 4: Particle flux to dose conversion factors: ICRP-21 for $\gamma H^{*} 10$ [6], ICRP-74 for neutron $H^{*} 10$ [7] and ASTM E-722 for silicon dose equivalent [8].

\subsection{Bulk shielding analysis}
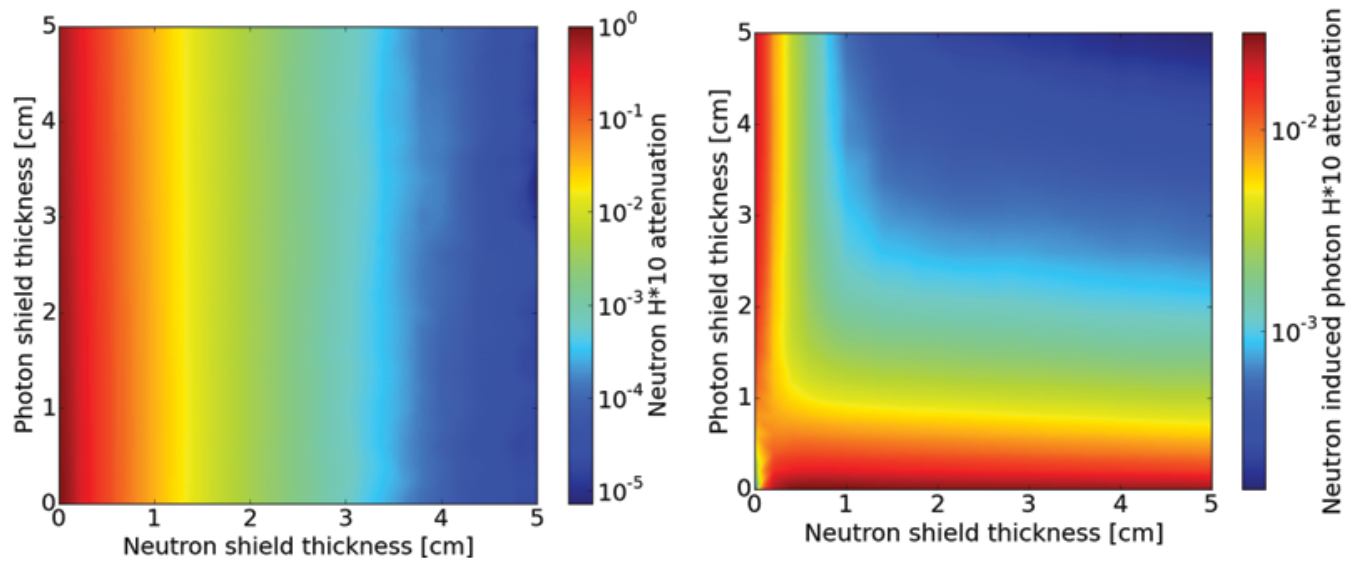

(a) Neutron $H^{*} 10$ dose attenuation vs. photon and neutron (b) Neutron induced photon $H^{*} 10$ dose attenuation shielding thickness.

vs. photon and neutron shielding thickness, normalized to incident neutron $H^{*} 10$ dose.

Figure 5: Color map of neutron and neutron induced photon $H^{*} 10$ dose attenuation vs. neutron and photon shielding thicknesses. Incident neutron spectrum (fig. 3a), $\frac{F}{T h}=0.1$, plane direct source. 
Firstly scoping calculations to identify the best shielding materials were performed on slab shields. Lead and tungsten were chosen as $\gamma$-ray shielding material and several boron or lithium loaded polyethylene-based materials as neutron shielding.

In fig. 5, that in case of incident neutrons, neutron shielding induces $\gamma$-radiation due to neutroninduced production of photons. This should be taken into account, as the values of neutron induced $\gamma$-ray dose might exceed that of neutrons at some shielding thicknesses combinations.

Since the irradiation tests were going on in parallel, some desired attenuation estimates based on the initial measurement data and identification of least radiation resistant components were made.

\subsection{Camera model design}

An initial stage camera model CAD design was proposed, which had the camera module, electronic and optics components modeled in. An exact transcript into MCNP [2] geometry was made utilizing a Rhino ${ }^{\circledR}$ Grasshopper plug-in script [11]. Each electronic component was tallied for neutron and photon flux and dose. In addition a mesh was superimposed across the model geometry (MCNP FMESH card), and both neutron and photon fluxes and doses calculated in each of the mesh voxels. Material compositions of the electronics and optics components were supplied by manufacturers and isotopic composition calculated using MATSSF [9].

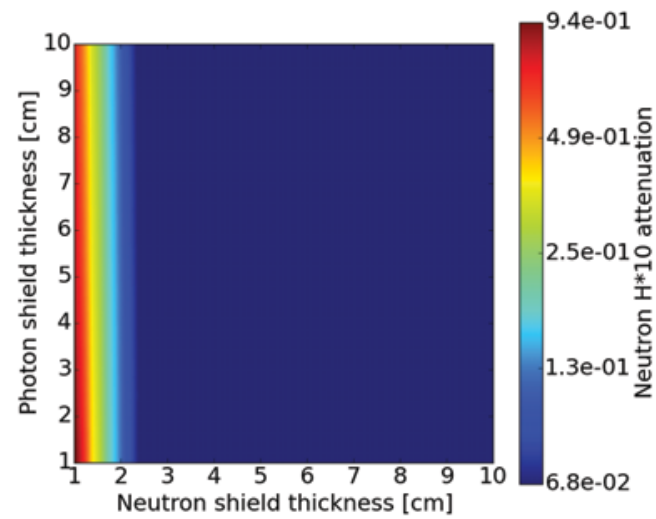

(a) Neutron $H^{*} 10$ dose attenuation on an internal component vs. photon and neutron shielding thickness.

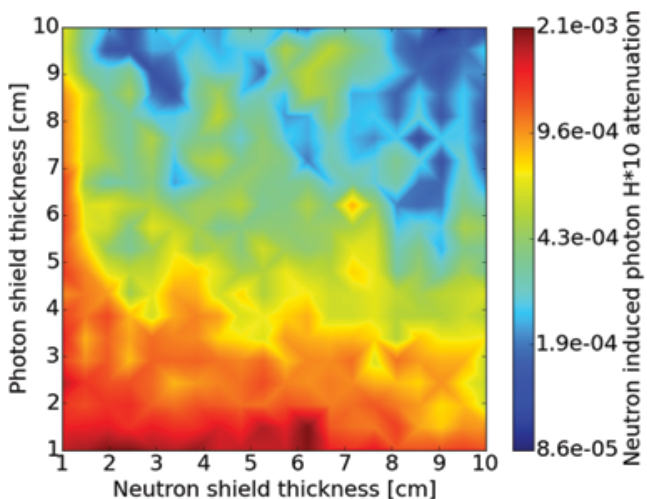

(b) Neutron induced photon $H^{*} 10$ dose attenuation on an internal component vs. photon and neutron shielding thickness, normalized to incident neutron $H^{*} 10$ dose.

Figure 6: Color plot of neutron and neutron induced photon $H^{*} 10$ dose attenuation on an internal component vs. neutron and photon shielding thicknesses. Incident neutron spectrum (fig. $3 \mathrm{a}), \frac{F}{T h}=$ 0.1 , plane direct source.

A parametric analysis was performed, varying thicknesses of both neutron and $\gamma$ shields, and neutron and photon source spectra and geometries. A Python v.2.7 script [12] (Python) was written in order to speed up the process and perform automatic input generation. Another, unshielded model was made, which served for normalization. It should be noted, that internal structure of the camera i.e. the components positions were changing during the parametric analysis, to accommodate several technical requirements, which in turn effect the shielding attenuation (figure $6 b$ ). 
Results would be presented in terms of attenuation vs. shielding thicknesses on color map-type graphs for each component, incident particle type and spectrum, source geometry and selected shielding materials (figure 6).

3D colored dose and flux maps were also produced from superimposed mesh results (fig: 7), to

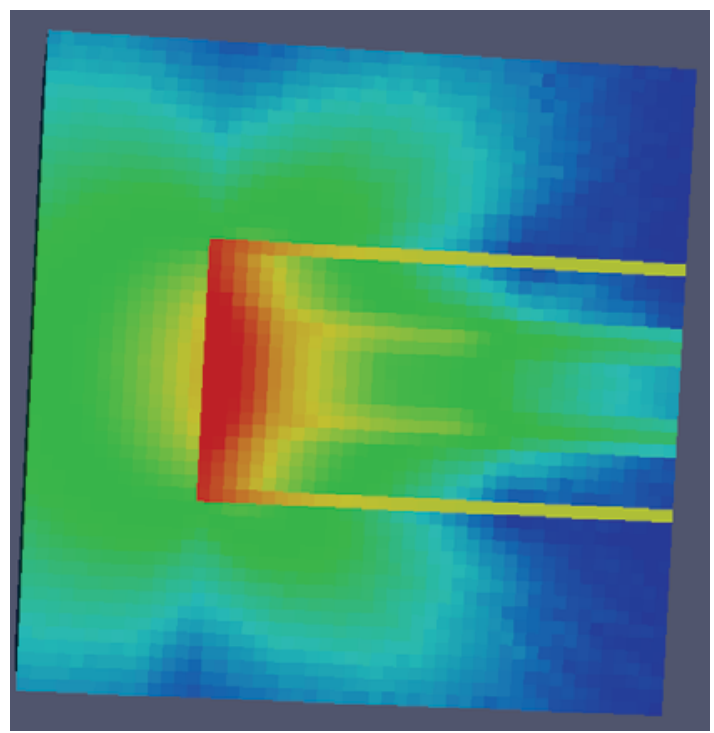

Figure 7: 3D ICRP-21 $H^{*} 10$ dose map, with incident ${ }^{60} \mathrm{Co}$ spectra, emitted from plane isotropic source.

asses the significant gradients, locate main contributors to the dose on components inside shielding and propose improvements. This enabled us, to place additional shielding to some components, that could not be relocated.

Using the obtained results, one can assess the shielding thicknesses and shielding material combinations, and a narrower shielding thickness variation with all the different source spectra and geometries was performed and dose attenuation values tallied electronic components and modules (items on figure 8). An optimal shielding thickness was established this way, and minor position changes and shielding thickness changes on key positions were suggested, to conform with the requirements.

It is interesting to note, that in case of reactor $\gamma$-ray spectra, the doses inside the shielding enclosure are up to more then $30 \%$ higher comparing to ${ }^{60} \mathrm{Co}$ spectrum. This is important, as the electronic components are commonly tested in ${ }^{60} \mathrm{Co}$ irradiation facilities, where test $\gamma$-spectra might significantly differ from $\gamma$-spectra during its intended operation, leading to an underestimation of shielding requirements (fig. 8).

\section{Summary}

When designing shielded radiation resistant electronic, a three step approach is proposed. Firstly the radiation tolerance of individual components should be determined, preferably by experiments in representative environment with respect to the intensity and spectrum of the incident particles, in our case gamma rays and neutrons Then the environmental source should be determined, i.e. the type of particles, their spectrum, angular flux, flux intensity, dose rate etc. When these boundary conditions 


\begin{tabular}{|c|c|c|c|c|c|c|c|}
\hline $\begin{array}{l}\text { Plane, direct } \\
\text { TRIGA MTK + 0.9 6,7 MeV }\end{array}$ & 0.527 & 0.547 & 0.546 & 0.547 & 0.545 & 0.665 & 0.483 \\
\hline $\begin{array}{l}\text { Plane, direct } \\
\text { TRIGA MTK + 0.1 6,7 MeV }\end{array}$ & 0.527 & 0.546 & 0.546 & 0.547 & 0.545 & 0.665 & 0.483 \\
\hline $\begin{array}{l}\text { Plane, direct } \\
\text { TRIGA MTK }\end{array}$ & 0.523 & 0.551 & 0.552 & 0.551 & 0.550 & 0.670 & 0.484 \\
\hline $\begin{array}{l}\text { Plane, direct } \\
\text { Cs-137 }\end{array}$ & 0.390 & 0.446 & 0.444 & 0.445 & 0.441 & 0.600 & 0.443 \\
\hline $\begin{array}{l}\text { Plane, direct } \\
\text { Co-60 }\end{array}$ & 0.474 & 0.530 & 0.529 & 0.529 & 0.526 & 0.651 & 0.461 \\
\hline $\begin{array}{l}\text { Plane, uniform over angle } \\
\text { TRIGA MTK + 0.9 6,7 MeV }\end{array}$ & 0.406 & 0.449 & 0.446 & 0.443 & 0.433 & 0.489 & 0.524 \\
\hline $\begin{array}{l}\text { Plane, uniform over angle } \\
\text { TRIGA MTK + 0.1 6,7 MeV }\end{array}$ & 0.406 & 0.449 & 0.446 & 0.443 & 0.433 & 0.489 & 0.524 \\
\hline $\begin{array}{l}\text { Plane, uniform over angle } \\
\text { TRIGA MTK }\end{array}$ & 0.406 & 0.448 & 0.446 & 0.442 & 0.432 & 0.488 & 0.522 \\
\hline $\begin{array}{l}\text { Plane, uniform over angle } \\
\text { Cs- } 137\end{array}$ & 0.277 & 0.321 & 0.319 & 0.316 & 0.306 & 0.386 & 0.440 \\
\hline $\begin{array}{l}\text { Plane, uniform over angle } \\
\text { Co-60 }\end{array}$ & 0.355 & 0.403 & 0.400 & 0.397 & 0.386 & 0.444 & 0.480 \\
\hline $\begin{array}{l}\text { Spherical } \\
\text { TRIGA MTK + 0.9 6,7 MeV }\end{array}$ & 0.436 & 0.429 & 0.428 & 0.425 & 0.415 & 0.460 & 0.498 \\
\hline $\begin{array}{l}\text { Spherical } \\
\text { TRIGA MTK + 0.1 6,7 MeV }\end{array}$ & 0.436 & 0.429 & 0.428 & 0.425 & 0.415 & 0.460 & 0.498 \\
\hline $\begin{array}{l}\text { Spherical } \\
\text { TRIGA MTK }\end{array}$ & 0.435 & 0.428 & 0.428 & 0.425 & 0.415 & 0.460 & 0.498 \\
\hline $\begin{array}{l}\text { Spherical } \\
\text { Cs-137 }\end{array}$ & 0.308 & 0.302 & 0.302 & 0.299 & 0.290 & 0.352 & 0.383 \\
\hline $\begin{array}{l}\text { Spherical } \\
\text { Co-60 }\end{array}$ & 0.388 & 0.382 & 0.382 & 0.378 & 0.368 & 0.418 & 0.452 \\
\hline & Item 1 & Item 2 & Item 3 & Item 4 & Item 5 & Item 6 & Item 7 \\
\hline
\end{tabular}

Figure 8: Kerma attenuation factors on items within the shielding enclosure, for different incident $\gamma$ spectra and source geometries. Differences in attenuation between reactor, ${ }^{60} \mathrm{Co}$ and ${ }^{137} \mathrm{Cs}$ (latter two commonly used for testing) $\gamma$ spectra may underestimate required shielding thicknesses.

are determined, the shielding design should be performed. In these activities computational support by Monte Carlo particle transport methods is essential. As the radiation field can change significantly from utility to utility all possible scenarios with respect to particles spectra and angular distribution are taken into account. For each of the scenarios the attenuation factor for the shield is calculated. It is important to note that $\gamma$ dose due to neutron induced $\gamma$-rays in the shield can be significantly higher than the environmental gamma ray dose, hence special attention should be paid to neutron field determination, which is often difficult [13]. As $\gamma$ radiation tolerance tests are commonly performed in dedicated facilities with ${ }^{137} \mathrm{Cs}$ or ${ }^{60} \mathrm{Co}$ sources, it is important to be aware that reactor $\gamma$ spectrum leads to approx $30 \%$ higher Si kerma at the same $H^{*} 10$ environmental $\gamma$ dose. One should also pay attention to dose definitions and measurement. $H^{*} 10$ environment dose is commonly measured with probes, the damage on electronics however is commonly measured with RADFETs for $\gamma$-rays and Si PIN diodes for neutrons [14]. Their response is proportional to Si kerma for $\gamma$-rays and damage function [8] for neutrons. The differences and relations between different doses, measurements and 
environment should be considered and the most practical approach to asses this is by Monte Carlo neutron and photon transport modeling as presented in the paper.

\section{References}

[1] ESCIES:European Space Components Information Exchange System, https://escies.org/ webdocument/showArticle?id=228\&groupid=6, accessed: 2016-06-14

[2] T. Goorley, M. James, T. Booth, F. Brown, J. Bull, L. Cox, J. Durkee, J. Elson, M. Fensin, R. Forster et al., Nuclear Technology 180, 298 (2012)

[3] L. Snoj, G. Žerovnik, A. Trkov, Applied Radiation and Isotopes 70, 483 (2012)

[4] K. Ambrožič, L. Snoj, Modeling of $\gamma$ field around irradiated TRIGA fuel elements by R2S method, in Proceedings of the ICRS-13 $\mathcal{E}$ RPSD-2016 (2016)

[5] K. Ambrožič, Master's thesis, Fakulteta za Matematiko in Fiziko, UL (2015)

[6] J. Hale, Radiology 111, 716 (1974)

[7] I.C. on Radiological Protection, I.C. on Radiation Units, Measurements, ICRP Publication 74: Conversion Coefficients for Use in Radiological Protection Against External Radiation, Annals of the ICRP (SAGE Publications, 1997), ISBN 9780080427393

[8] A. International, ASTM E722, ASTM International, West Conshohocken, Philadelphia, USA (2014)

[9] A. Trkov, G. Žerovnik, L. Snoj, M. Ravnik, Nuclear Instruments and Methods in Physics Research A 610, 553 (2009)

[10] M. Chadwick, P. Obložinsky, M. Herman, N. Greene, R. McKnight, D. Smith, P. Young, R. MacFarlane, G. Hale, S. Frankle et al., Nuclear Data Sheets 107, 2931 (2006), evaluated Nuclear Data File ENDF/B-VII.0

[11] B. Kos, L. Snoj, Master's thesis, Fakulteta za matematiko in fiziko, Univerza v Ljubljani (2015)

[12] G. Rossum, Tech. rep., Amsterdam, The Netherlands, The Netherlands (1995)

[13] I. Remec, M. Najžer, G. Pregl, Ph.D. thesis (1991), https: //books.google.si/books?id=QnVhOQAACAAJ

[14] F. Ravotti, Ph.D. thesis, Université Montpellier II (2006) 